\title{
A case of multiple synchronous lung adenocarcinomas with differing EGFR mutations
}

\author{
Hideomi Hasegawa $\cdot$ Hideki Kimura $\cdot$ \\ Masato Shingyoji • Makiko Itami · Akinobu Araki • \\ Sana Yokoi · Hajime Kageyama · Toshihiko Iizasa
}

Received: 10 December 2012/ Accepted: 4 March 2013/Published online: 28 March 2013

(C) The Japan Society of Clinical Oncology 2013

\begin{abstract}
We report a case of four synchronous lung cancers, each with a different epidermal growth factor receptor (EGFR) mutation in the tyrosine kinase domain. Bilateral double lung cancer was diagnosed clinically, and a left upper lobectomy and partial left S6 resection were performed. Right S1 segmental resection was performed later. Pathological examination revealed three lung adenocarcinomas in the left lung (S1 $+2, \mathrm{~S} 3$, and $\mathrm{S} 6)$ and one in the right. Three different mutations of EGFR were identified with the polymerase chain reaction and direct sequencing of DNA from the three left lung tumors. The EGFR mutation on the right also differed from that of the left lung tumors.
\end{abstract}

Keywords Lung cancer - Epidermal growth factor receptor (EGFR) · Surgery · Adenocarcinoma

\section{Introduction}

Lung adenocarcinoma is one of the leading causes of death in Japan. Advances in high-resolution computed tomography (CT) and the prevalence of CT screening for lung cancer have increased the detection of multiple solid and ground-glass opacity (GGO) nodules, which are often atypical adenomatous hyperplasia (AAH), bronchioloalveolar

H. Hasegawa $(\bowtie)$

Chiba Cardiovascular Center, 575 Tsurumai, Ichihara,

Chibaken 290-0512, Japan

e-mail: hijowomi@gmail.com

H. Kimura - M. Shingyoji - M. Itami - A. Araki - S. Yokoi ·

H. Kageyama · T. Iizasa

Chiba Cancer Center, 666-2 Nitona, Chuuouku, Chiba,

Chibaken 260-0801, Japan carcinoma (BAC) and adenocarcinoma [1]. The multifocality of lung nodules may be due to either the dissemination of malignant cells from a single primary tumor (intrapulmonary spread) or synchronous development of multifocal independent lesions. The distinction of intrapulmonary metastases from independent primary tumors is of great clinical importance as it influences the staging and therapeutic strategy $[2,3]$. We report a case of four synchronous lung adenocarcinomas in the lungs. Gene mutation analysis showed these tumors to be independent, genetically differing lesions with differing epidermal growth factor receptor (EGFR) mutations. Molecular analysis in addition to histological diagnosis of the clonal relationships between multiple lesions helps in the differentiation between metastases and multifocal lung cancers [4].

\section{Case presentation}

A 55-year-old woman with a history of smoking (Brinkman index: 500) and breast cancer was referred to our hospital for abnormal shadows in the upper lobes of both lungs (Fig. 1). Bronchoscopic examination with endobronchial ultrasound trans-bronchial needle aspiration (EBUSTBNA) [5] and positron emission tomography indicated lung cancer of both upper lobes without lymph node involvement or distant metastasis. Double primary lung cancer was diagnosed in both lungs, and surgery was performed. The first operation was performed on the left lung, and the right lung procedure followed 2 months later.

The left $\mathrm{S} 1+2 \mathrm{~b}$ tumor, which measured up to $2.8 \mathrm{~cm}$ in diameter, was adenocarcinoma with mixed subtype (non-mucinous BAC $>$ papillary adenocarcinoma: Fig. 2). In the course of a left upper lobectomy, S6 partial resection was also performed because a small nodule, which had not 
Fig. 1 Chest radiograph and computed tomogram showing abnormal shadows in the upper lobes (arrows) of both lungs
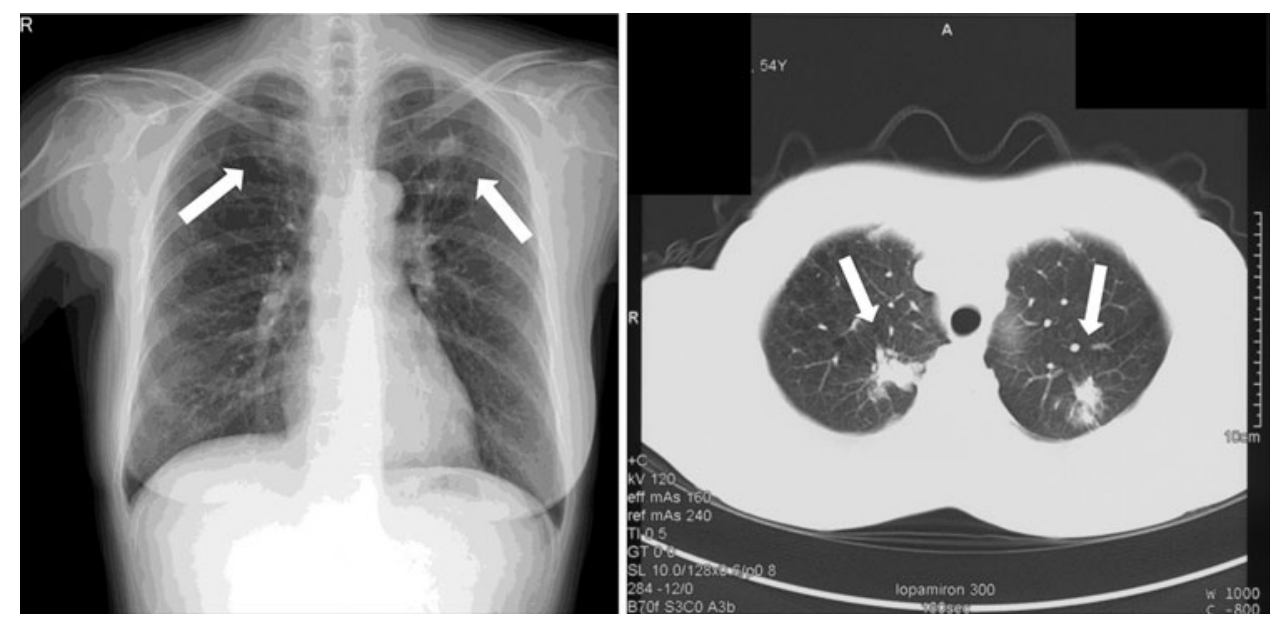

Fig. 2 The left $\mathrm{S} 1+2 \mathrm{~b}$ tumor, measuring $2.8 \mathrm{~cm}$ in diameter a was an adenocarcinoma with a mixed subtype: non-mucinous BAC $>$ papillary adenocarcinoma (hematoxylin $\&$ eosin staining). b At low magnification $(\times 100)$ and $\mathbf{c}$ at high magnification $(\times 200)$
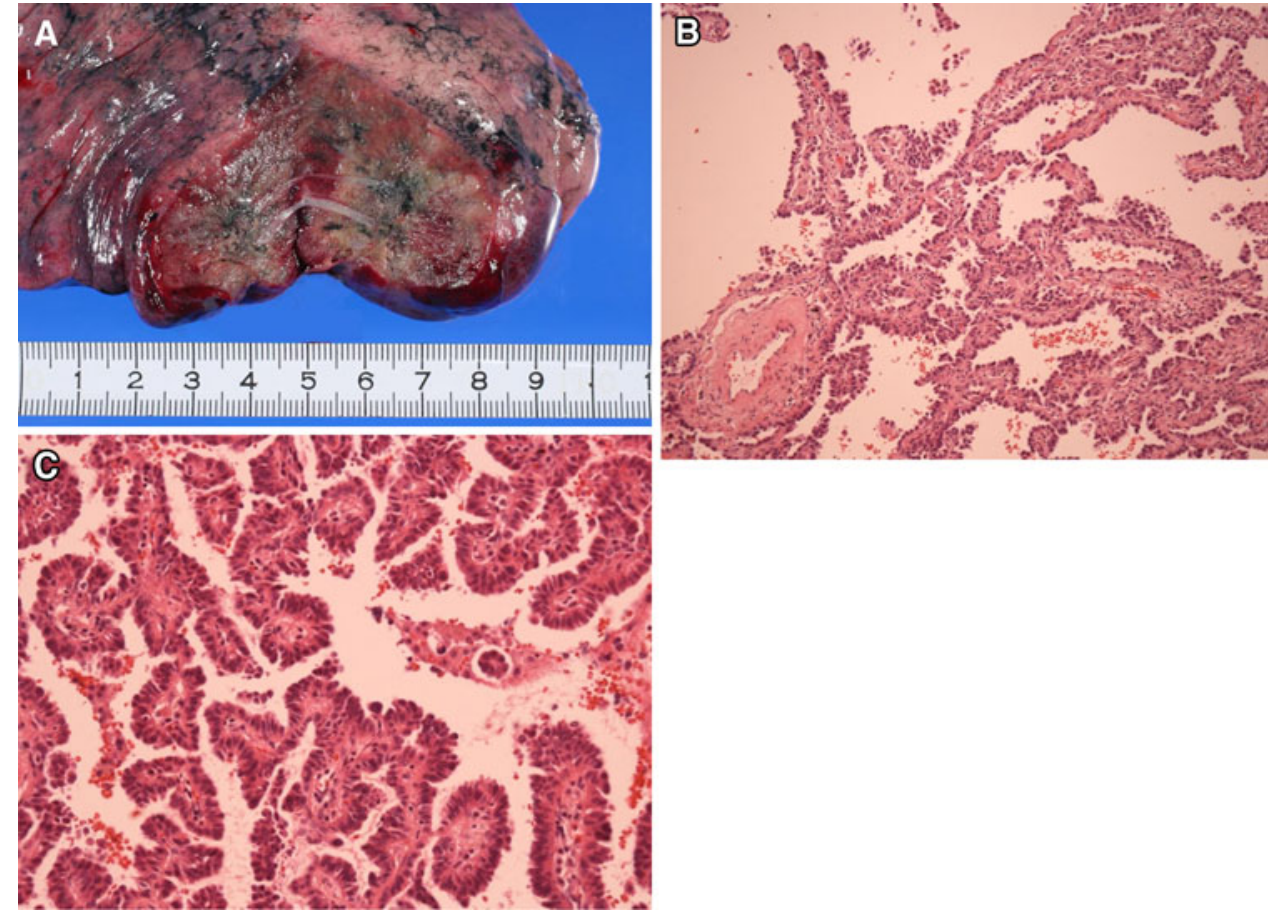

been detected by preoperative CT scan, was detected on palpation of the left lower lobe. A 17-mm S6 tumor was also adenocarcinoma combined with a subtype (nonmucinous BAC $>$ papillary adenocarcinoma: Fig. 3). The third tumor, in the left $\mathrm{S} 3$, which measured $0.8 \mathrm{~cm}$ in diameter, was revealed during the pathological examination of the left upper lobe and was identified as bronchioloalveolar carcinoma (BAC: Fig. 4). Right S1 segmentectomy was performed 2 months later. The fourth tumor, which measured $2.4 \mathrm{~cm}$ in diameter, was an adenocarcinoma with a mixed subtype (papillary $\gg$ nonmucinous BAC: Fig. 5).

We performed DNA extraction and analyzed EGFR mutations at exons 18-21 [6] and K-ras mutations at codons 12, 13 and 61 [7] using the nested polymerase chain reaction (PCR). DNA was extracted from the tissues with an Allprep DNA/RNA/Protein Mini Kit (QIAGEN) according to the manufacturer's instructions. DNA concentration and purity were determined using UV spectrophotometry (Nanodrop). Exons 18, 19, 20 and 21 of the EGFR were sequentially amplified by two rounds of PCR and were subjected to direct sequencing. There was no $\mathrm{K}$-ras mutation in the four lesions, but the $\mathrm{S} 1+2 \mathrm{~b}$ tumor had point mutations on exon 21 (L858R) and exon 18 (E709A, Table 1; Fig. 6). The left S6 tumor had a mutation on exon 20 (D770-N771 insD) and another on S3 tumor exon 20 (S768I). Right S1 adenocarcinoma had mutations on exon 18 (G719C) and exon 20 (S768I) (Table 1; Fig. 6). 
Fig. 3 Left S6 tumor $17 \mathrm{~mm}$ in diameter. a Adenocarcinoma with a mixed subtype: nonmucinous BAC > papillary adenocarcinoma (hematoxylin $\&$ eosin staining). b At low magnification $(\times 100)$ and $\mathbf{c}$ at high magnification $(\times 200)$
Fig. 4 Left S3 tumor was revealed during the pathological examination of the left upper lobe and was diagnosed as bronchioloalveolar carcinoma (hematoxylin \& eosin staining) a at low magnification $(\times 100)$ and $\mathbf{b}$ at high magnification $(\times 200)$
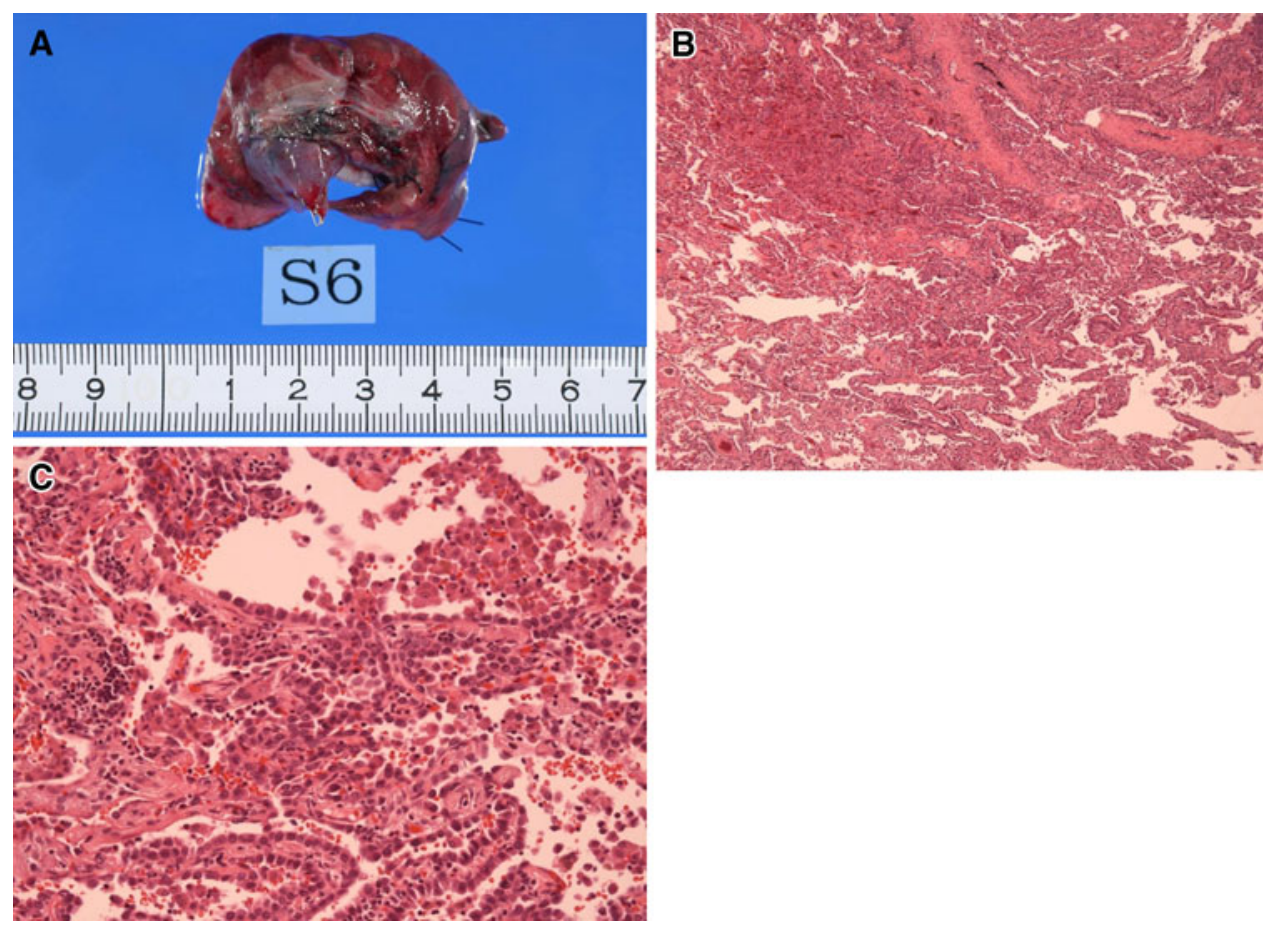

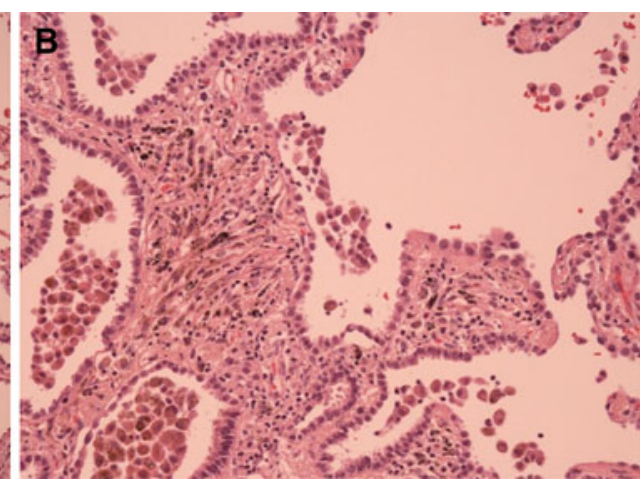

Fig. 5 Right lung tumor measuring $2.4 \mathrm{~cm}$ in diameter: adenocarcinoma $\mathbf{a}$ with a mixed subtype: papillary $\gg$ nonmucinous BAC (hematoxylin \& eosin staining) b at low magnification $(\times 100)$ and $\mathbf{c}$ at high magnification $(\times 200)$
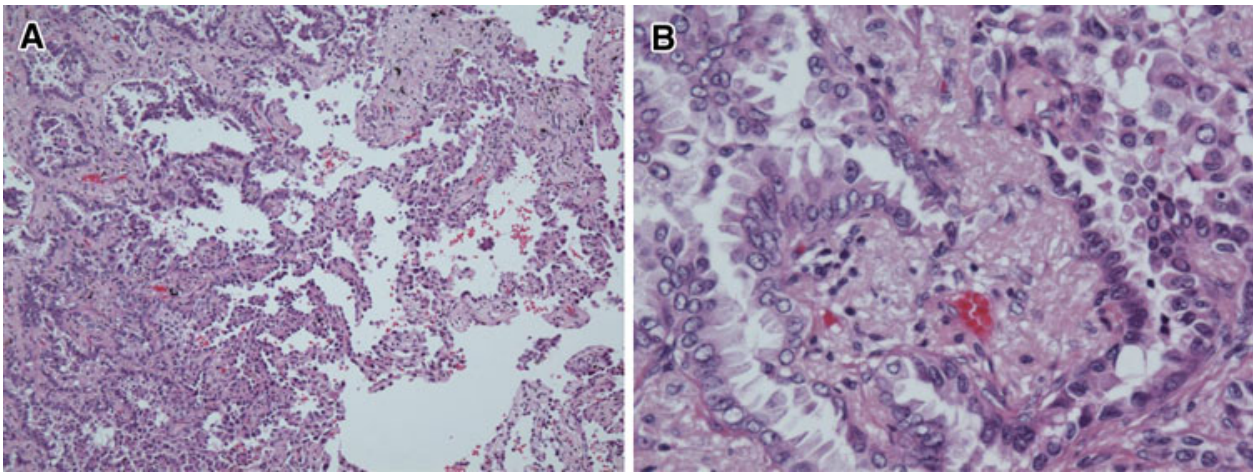

\section{Discussion}

Molecular analysis of these multifocal lesions indicated that they arose as independent events rather than through intrapulmonary expansion or metastasis. However, we cannot contend that all the lung nodules are of independent origin because the use of EGFR mutational status as a surrogate maker of clonality has some limitations [8]. A metastatic tumor may appear different from the primary tumor at the genetic level because progressive accumulation of genetic 
Table 1 EGFR mutations of each tumor

\begin{tabular}{lll}
\hline Tumor location & Pathological type & EGFR mutation \\
\hline Left S1 +2 & Adenocarcinoma with mixed subtype & L858R (exon 21) \\
& & E709A (exon 18) \\
Left S3 & Bronchioloalveolar carcinoma & S768I (exon 20) \\
Left S6 & Adenocarcinoma with mixed subtype & D770-N771 (insD) (exon 20) \\
Right S1 & Adenocarcinoma with mixed subtype & G719C (exon 18) \\
\hline
\end{tabular}

analysis by direct sequencing of the four lesions

\begin{tabular}{|c|c|c|c|c|c|c|c|c|c|c|c|c|c|c|c|c|c|c|c|c|c|c|}
\hline $\mathrm{T}$ & G & G & G & C & G & G & G & $\mathrm{C}$ & $\mathrm{CA}$ & A & $\mathrm{T}$ & G & G & $\mathrm{C}$ & C A & G & C & G & $T$ & G & G & \\
\hline 101 & & 103 & & 105 & & 107 & & 109 & 111 & & 24 & 125 & 126 & 127 & 12129 & 130 & 131 & 132 & 1331 & 1341 & 135 & 13 \\
\hline
\end{tabular}
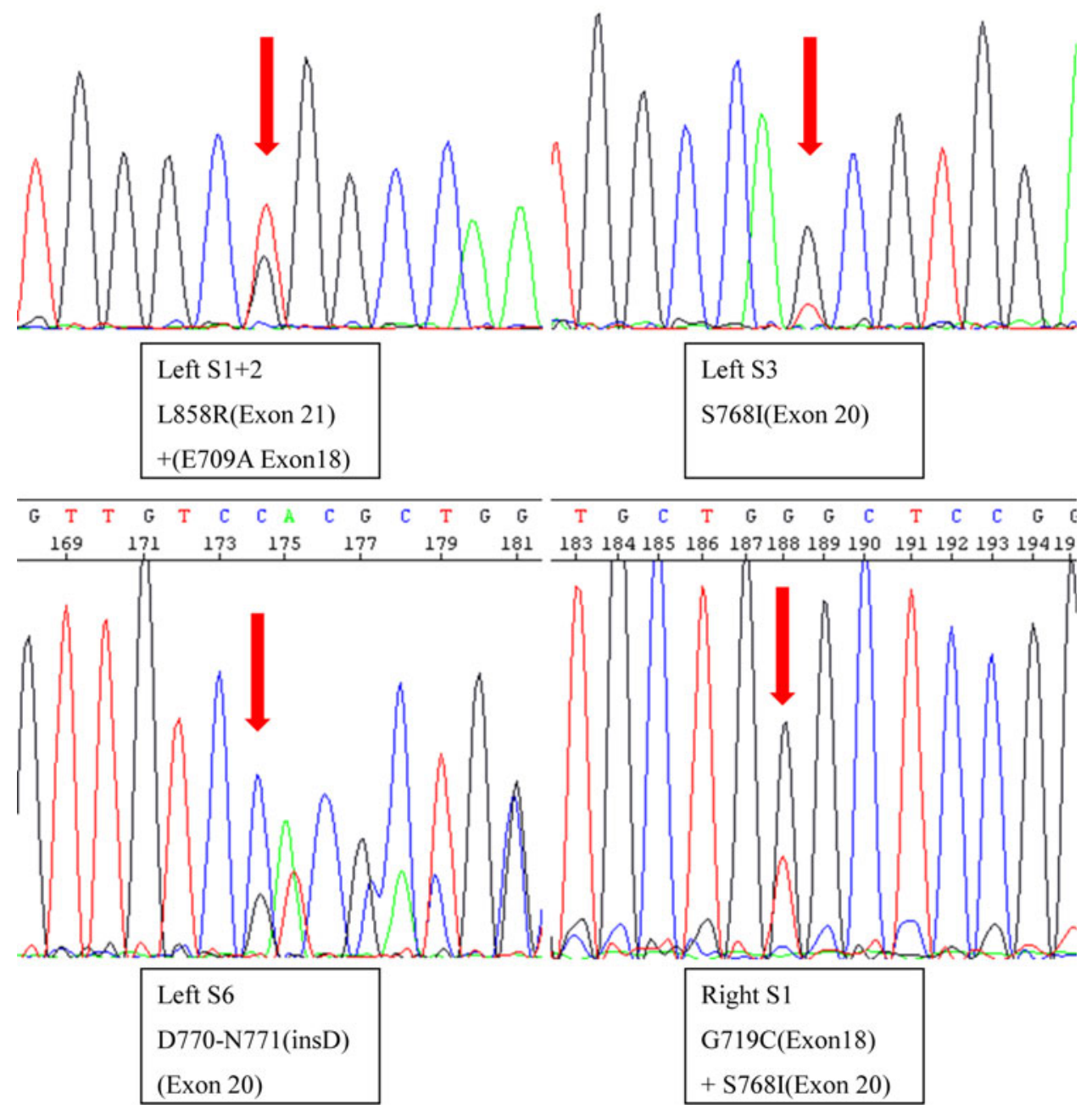

alteration is not uncommon in metastases [9]. Thus, analysis of the genetic alterations could be useful for identifying clonality in the early stages of the tumorigenesis and must be used together with a histological diagnosis. From the histological point of view, the four lesions presented in this report are synchronous primary carcinomas in the early stages of carcinogenesis because they are all: (1) moderately to well- differentiated adenocarcinomas, (2) accompanied by BAC on the periphery of the lung or with central scar formation, (3) free of blood vessel or lymph vessel invasion and (4) free of lymph node metastasis. One of the mutations (exon 20: S768I) observed in the left S3 was identical with one in the right $\mathrm{S} 1$, but the $\mathrm{S} 1$ tumor had an another mutation in exon 18 (G719C). The results of molecular analysis suggested that 
the right $\mathrm{S} 1$ tumor may have been a metastasis from the left S3 BAC. From a histological point of view, however, it is hard to accept that the right $\mathrm{S} 1$ tumor, $2.4 \mathrm{~cm}$ in diameter with a solid papillary pattern, metastasized from the left $\mathrm{S} 3$ BAC with a pure GGO of $0.8 \mathrm{~cm}$ in diameter.

Although we have often encountered multiple lung lesions, many of which are intrapulmonary metastases [10], synchronous or metachronous double cancers are not uncommon in the lung, but three or more synchronous multifocal cancers are rare. Furthermore, four synchronous primary cancers with different EGFR mutations in the same patient are exceedingly rare and, to the best of our knowledge, may be the first ever described. Chung et al. [8] reported patients with multiple lung nodules presented as GGO and investigated somatic mutations of EGFR and K-ras. They presented two cases of adenocarcinoma with different types of EGFR mutation (exon 19 del and exon 21: L858R) in a group of 24 patients with multifocal lung lesions. Ikeda et al. [1] reported 26 cases with multicentric lung adenocarcinoma and atypical adenomatous hyperplasias and 4 cases of adenocarcinoma or BAC with differing EGFR mutations. Takamochi et al. [11] reported on 36 patients with multifocal adenocarcinoma to clarify the clonality status based on the mutation patterns of EGFR and K-ras. Five out of these 36 cases showed different EGFR mutation patterns. In the same report, the authors described a case in which there were four adenocarcinoma lesions, three of which had the same EGFR mutation pattern (exon 21: L858R). Bell et al. [12] reported a family with multiple cases of non-small cell lung cancer associated with germ line transmission of the EGFR T790M mutation. A 50-year-old member of this family who had a history of smoking presented with bilateral multifocal noncalcified nodules. Molecular analysis of five independent BACs obtained by surgery indicated the T790M mutation in all tumor specimens in addition to the germ line mutation in the peripheral blood mononuclear cells. Three of the lesions had additional somatic activating mutations in EGFR (L858R in two cases and delL747-751 in the third). Although this T790M mutation was not observed in our case, a similar mechanism inducing multifocal lung adenocarcinoma by an another germ line mutation may have contributed to the carcinogenesis in this case.

Acknowledgments We thank Mr. C.W.P. Reynolds, associated with the Department of International Medical Communications, Tokyo Medical University, for his careful revision of the English of this manuscript. This work was supported in part by a non-profit organization: Chiba Clinical Cancer Support Organization (CCCSO).

Conflict of interest The authors state no conflict of interest.

\section{References}

1. Ikeda K, Nomori H, Ohba Y, Shibata H, Mori T, Honda Y, Iyama K, Kobayashi T (2008) Epidermal growth factor receptor mutations in multicentric lung adenocarcinomas and atypical adenomatous hyperplasias. J Thorac Oncol 3:467-471

2. Girard N, Deshpande C, Lau C, Finley D, Rusch V, Pao W, Travis WD (2009) Comprehensive histologic assessment helps to differentiate multiple lung primary nonsmall cell carcinomas from metastases. Am J Surg Pathol 33:1752-1764

3. Tanner NT, Pastis NJ, Sherman C, Simon GR, Lewin D, Silvestri GA (2012) The role of molecular analyses in the era of personalized therapy for advanced NSCLC. Lung Cancer 76:131-137

4. Gallegos Ruiz MI, van Cruijsen H, Smit EF, Grünberg K, Meijer GA, Rodriguez JA, Ylstra B, Giaccone G (2007) Genetic heterogeneity in patients with multiple neoplastic lung lesions: a report of three cases. J Thorac Oncol 2:12-21

5. Nakajima T, Yasufuku K, Nakagawara A, Kimura H, Yoshino I (2011) Multigene mutation analysis of metastatic lymph nodes in non-small-cell lung cancer diagnosed by endobronchial ultrasound-guided transbronchial needle aspiration. Chest 140:13191324

6. Nakano H, Soda H, Nakamura Y, Uchida K, Takasu M, Nakatomi K, Izumikawa K, Hayashi T, Nagayasu T, Tsukamoto K, Kohno $S$ (2007) Different epidermal growth factor receptor gene mutations in a patient with 2 synchronous lung cancers. Clin Lung Cancer 8:562-564

7. Sharma SV, Bell DW, Settleman J, Haber DA (2007) Epidermal growth factor receptor mutations in lung cancer. Nat Rev Cancer 7:169-181

8. Chung JH, Choe G, Jheon S, Sung SW, Kim TJ, Lee KW, Lee JH, Lee CT (2009) Epidermal growth factor receptor mutation and pathologic-radiologic correlation between multiple lung nodules with ground-glass opacity differentiates multicentric origin from intrapulmonary spread. J Thorac Oncol 4:1490-1495

9. Wang X, Wang M, MacLennan GT, Abdul-Karim FW, Eble JN, Jones TD, Olobatuyi F, Eisenberg R, Cummings OW, Zhang S, Lopez-Beltran A, Montironi R, Zheng S, Lin H, Davidson DD, Cheng L (2009) Evidence for common clonal origin of multifocal lung cancers. J Natl Cancer Inst 101:560-570

10. Sepehripour AH, Nasir A, Shah R (2012) Multiple synchronous primary tumours in a single lobe. Interact Cardiovasc Thorac Surg 14:340-341

11. Takamochi K, Oh S, Matsuoka J, Suzuki K (2012) Clonality status of multifocal lung adenocarcinomas based on the mutation patterns of EGFR and K-ras. Lung Cancer 75:313-320

12. Bell DW, Gore I, Okimoto RA, Godin-Heymann N, Sordella R, Mulloy R, Sharma SV, Brannigan BW, Mohapatra G, Settleman J, Haber DA (2005) Inherited susceptibility to lung cancer may be associated with the T790M drug resistance mutation in EGFR. Nat Genet 37:1315-1316 